\title{
TOPOLOGICAL LIFSHITZ PHASE TRANSITION IN EFFECTIVE MODEL OF QCD WITH CHIRAL SYMMETRY NON-RESTORATION
}

\author{
TRAN HUU PHAT \\ Vietnam Atomic Energy Institute, 59 Ly Thuong Kiet, Hanoi, Vietnam. \\ Center for Advance Study, Dong Do University, 170 Pham Van Dong, Hanoi, Vietnam \\ Email: \\ PHUNG THI THU HA \\ Institute of Physics, 10 Dao Tan, Hanoi, Vietnam \\ Vietnam Atomic Energy Institute, 59 Ly Thuong Kiet, Hanoi, Vietnam \\ Email: \\ NGUYEN TUAN ANH \\ Electric Power University, 235 Hoang Quoc Viet, Hanoi, Vietnam \\ Email: \\ Received 25 September 2013 \\ Accepted for publication 22 October 2013
}

\begin{abstract}
The topological Lifshitz phase transition is studied systematically within an effective model of QCD, in which the chiral symmetry, broken at zero temperature, is not restored at high temperature and/or baryon chemical potential. It is found that during phase transition the quark system undergoes a first-order transition from low density fully-gapped state to high density state with Fermi sphere which is protected by momentum-space topology. The Lifshitz phase diagram in the plane of temperature and baryon chemical potential is established. The critical behaviors of various equations of state are determined.
\end{abstract}

\section{INTRODUCTION}

Quantum Chromodynamics (QCD) is actually considered to be the theory of strongly interacting systems of quarks and gluons. At finite baryon chemical potential the theory also provides information on nuclear properties. We have expected that QCD at finite temperature $T$ and baryon chemical potential $\mu$ has a very rich phase structure [1] such as the chiral symmetry restoration at high temperature and/or baryon chemical potential, the thermal phase transition from the Nambu-Goldstone phase to quark - gluon plasma, the phase transition from the Nambu-Goldstone phase to the color superconductivity at low $T$ and so on. The experimental exploration of these phase transitions is being actively carried out in RHIC (Relativistic Heavy Ion Collider) and then in LHC (Large Hadron Collider). Especially, the quantum phase transition from Nambu-Goldstone phase to the color superconductivity at low $T$, which is of interest in the interiors of neutron 
stars and possible quark stars, is also relevant for experimental realizations with moderate energy heavy-ion collisions. In addition to these phase transition patterns, based on the fundamental works of Weinberg [2], Dolan and Jackiw [3] one believes that generally symmetry will get restored at high temperature from the broken phase at $T=0$. However, in reality there exist physical systems which manifest the symmetry non-restoration (SNR) at high temperature. They associate with numerous different materials [4]. The theoretical calculations performed in Refs. $[5,6]$ proved that SNR really occurs in several models. The physical significance of these phenomena rests in the fact that SNR may have remarkable consequences for cosmology, namely, within the scenario of SNR the puzzle relating to topological defects in the Standard Cosmological Big Bang of universe could be solved $[5,6]$. Concerning the chiral symmetry of QCD the calculations derived from [7] showed that the chiral symmetry is not restored at the deconfining temperature in the case when $\operatorname{Re}\left[\operatorname{tr}_{c}(P)\right]<0$, here $P$ is the Polyakov loop; this agrees with the simulation study [8]. Until now the phase structure of QCD has been step by step established by mean of either Lattice QCD [9] or effective models of QCD for the scenario of chiral symmetry restoration at high temperature and/or baryon density. Nevertheless, there remains lacking information on phase structure of QCD corresponding to non-restoration scenario.

In view of what presented above, the present paper aims at remedying this gap, namely, we focus on investigating QPT's of QCD associating with the sector of chiral symmetry non-restoration. For this purpose, we start from an effective model, whose Lagrangian reads

$$
L=\bar{\psi}\left[\gamma^{\mu}\left(i \partial_{\mu}-g_{v} \omega_{\mu}\right)-m_{q}+g_{s}\left(\phi+i \gamma^{5} \vec{\tau} \cdot \vec{\pi}\right)\right] \psi+\frac{1}{2}\left(\partial_{\mu} \phi \partial^{\mu} \phi+\partial_{\mu} \vec{\pi} \partial^{\mu} \vec{\pi}\right)-\frac{1}{4} F_{\mu \nu} F^{\mu \nu}-U,
$$

where

$$
\begin{aligned}
U & =-\frac{1}{2} g_{s v}\left(\phi^{2}+\vec{\pi}^{2}\right) \omega_{\mu} \omega^{\mu}+\frac{m^{2}}{2}\left(\phi^{2}+\vec{\pi}^{2}\right)+\frac{\lambda}{4 !}\left(\phi^{2}+\vec{\pi}^{2}\right)^{2}, \\
\lambda & =\frac{3}{f_{\pi}^{2}}\left(m_{\sigma}^{2}-m_{\pi}^{2}\right), \\
m^{2} & =\frac{1}{2}\left(3 m_{\pi}^{2}-m_{\sigma}^{2}\right) .
\end{aligned}
$$

In (1) $F_{\mu \nu}=\partial_{\mu} \omega_{\nu}-\partial_{\nu} \omega_{\mu} ; \psi, \phi, \vec{\pi}, \omega_{\mu}$ are respectively the field operators of quark, sigma meson, pion and omega meson; $m_{q}, m_{\sigma}, m_{\pi}$, and $m_{\omega}$ are respectively masses of current quark, sigma meson, pion and omega meson; $m, \lambda, g_{s}, g_{v}, g_{s v}$ are the model parameters.

The paper is organized as follows. The Section II is devoted to the calculation of effective potential and its related physical quantities. The chiral symmetry non-restoration is dealt with in Section III. In Section IV we focus on the study of Lifshitz phase transition. The conclusion and discussion are presented in Section V.

\section{EFFECTIVE POTENTIAL AND ITS RELATED PHYSICAL QUANTITIES}

Starting from the Lagrangian (1) let us calculate the effective potential and its related quantities. 
In the mean field approximation

$$
\langle\phi\rangle=u-\sigma_{0}, \quad\left\langle\pi_{i}\right\rangle=0 \quad\left\langle\omega_{\mu}\right\rangle=\omega_{0} \delta_{0 \mu},
$$

the Lagrangian (1) takes the form

$$
\mathcal{L}=\bar{\psi}\left[\gamma^{0}\left(p_{0}+\mu^{*}\right)+\vec{\gamma} \cdot \vec{p}-m_{q}^{*}\right] \psi-U
$$

in which

$$
\begin{aligned}
U & =-\frac{1}{2} m_{\omega}^{* 2} \omega^{2}+\frac{m^{2} m_{q}^{* 2}}{2 g_{s}^{2}}+\frac{\lambda m_{q}^{* 4}}{24 g_{s}^{4}}, \\
m_{q}^{*} & =m_{q}-g_{s} u, \quad m_{q}=g_{s} \sigma_{0}, \\
m_{\omega}^{*} & =m_{\omega}-g_{s v} u, \quad m_{\omega}=g_{s v} \sigma_{0}, \\
\mu^{*} & =\mu-g_{v} \omega_{0} .
\end{aligned}
$$

Effective potential derived from (2) reads

$$
\Omega=U-\frac{6}{\pi^{2}} \int p^{2} d p\left[E_{p}+T \ln \left(1+e^{-E_{+} / T}\right)+T \ln \left(1+e^{-E_{-} / T}\right)\right]
$$

where

$$
E_{ \pm}(\vec{p})=E_{p} \pm \mu^{*}, \quad E_{p}=\sqrt{\vec{p}^{2}+m_{q}^{* 2}}
$$

The ground state of the system is determined by minimizing (4)

$$
\frac{\partial \Omega}{\partial u}=0, \quad \frac{\partial \Omega}{\partial \omega_{0}}=0
$$

yielding

$$
\begin{aligned}
\frac{\partial U}{\partial u} & =g_{s} \rho_{s}, \quad \rho_{s}=\frac{6}{\pi^{2}} \int p^{2} d p \frac{m_{q}^{*}}{E_{p}}\left(n_{-}+n_{+}-1\right), \\
\frac{\partial U}{\partial \omega_{0}} & =g_{v} \rho_{v}, \quad \rho_{v}=\frac{6}{\pi^{2}} \int p^{2} d p\left(n_{-}-n_{+}\right), \quad n_{ \pm}=\left[1+e^{E_{ \pm} / T}\right]^{-1},
\end{aligned}
$$

the first of which is usually called the gap equation. Combining the gap equation and (3) gives

$$
g_{v}^{2} \frac{m_{q}^{2}}{m_{\omega}^{2} m_{q}^{* 3}} \rho_{v}^{2}-\frac{m^{2} m_{q}^{*}}{g_{s}^{2}}-\frac{\lambda m_{q}^{* 3}}{6 g_{s}^{4}}=\rho_{s} .
$$

Next let us establish the formulas for thermodynamic quantities, the first one among them is pressure $P$ to be determined through $\Omega$ taken at minimum

$$
P=-\Omega \text {. }
$$

The quark density is defined as

$$
\rho=\frac{\partial P}{\partial \mu}
$$

giving

$$
\rho=\rho_{v} .
$$


In term of quark density the expression for $P$ is written

$$
\begin{aligned}
P= & \frac{m^{2} m_{q}^{* 2}}{2 g_{s}^{2}}+\frac{\lambda m_{q}^{* 4}}{24 g_{s}^{4}}-g_{v}^{2} \frac{m_{q}^{2}}{2 m_{\omega}^{2} m_{q}^{* 2}} \rho_{v}^{2} \\
& +\frac{6}{\pi^{2}} \int p^{2} d p\left[E_{p}+T \ln \left(1+e^{-E_{+} / T}\right)+T \ln \left(1+e^{-E_{-} / T}\right)\right] .
\end{aligned}
$$

The energy density $\epsilon$ is then obtained by a Legendre of $P$

$$
\epsilon=-P+T s+\mu \rho
$$

which together with (8) provides

$$
\epsilon=\frac{m^{2} m_{q}^{* 2}}{2 g_{s}^{2}}+\frac{\lambda m_{q}^{* 4}}{24 g_{s}^{4}}+g_{v}^{2} \frac{m_{q}^{2}}{2 m_{\omega}^{2} m_{q}^{* 2}} \rho_{v}^{2}+\frac{6}{\pi^{2}} \int p^{2} d p E_{p}\left(n_{-}+n_{+}-1\right) .
$$

Here

$$
s=\frac{6}{\pi^{2}} \int p^{2} d p\left[n_{-} \ln n_{-}+\left(1-n_{-}\right) \ln \left(1-n_{-}\right)+n_{+} \ln n_{+}+\left(1-n_{+}\right) \ln \left(1-n_{+}\right)\right] .
$$
eration.

Eqs. (8) and (9) are the basic equations of state of the quark system under consid-

At zero temperature Eqs. (6), (7), (8) and (9) reduce to

$$
\begin{aligned}
& g_{v}^{2} \frac{m_{q}^{2}}{m_{\omega}^{2} m_{q}^{* 3}} \rho^{2}-\frac{m^{2} m_{q}^{*}}{g_{s}^{2}}-\frac{\lambda m_{q}^{* 3}}{6 g_{s}^{4}}=\frac{6}{\pi^{2}} \int_{0}^{p_{F}} p^{2} d p \frac{m_{q}^{*}}{E_{p}} \\
& \rho=\frac{6 p_{F}^{3}}{3 \pi^{2}} \\
& P=\frac{m^{2} m_{q}^{* 2}}{2 g_{s}^{2}}+\frac{\lambda m_{q}^{* 4}}{24 g_{s}^{4}}-g_{v}^{2} \frac{m_{q}^{2}}{2 m_{\omega}^{2} m_{q}^{* 2}} \rho^{2}+\frac{6}{\pi^{2}} \int_{0}^{p_{F}} p^{2} d p E_{p}, \\
& \epsilon=\frac{m^{2} m_{q}^{* 2}}{2 g_{s}^{2}}+\frac{\lambda m_{q}^{* 4}}{24 g_{s}^{4}}+g_{v}^{2} \frac{m_{q}^{2}}{2 m_{\omega}^{2} m_{q}^{* 2}} \rho^{2}+\frac{6}{\pi^{2}} \int_{0}^{p_{F}} p^{2} d p E_{p} .
\end{aligned}
$$

With the aid of the analytical expressions derived in the foregoing Section let us investigate successively the following phase transitions.

\section{CHIRAL SYMMETRY NON-RESTORATION}

To calculate numerically we use inputs such as $g_{s}=3.3 \mathrm{fm}^{2}, g_{v}=0.99 \mathrm{fm}^{2}, m_{q}=5$ $\mathrm{MeV}, m_{\sigma}=600 \mathrm{MeV}, m_{\omega}=783 \mathrm{MeV}$.

Let us first numerically compute the $T$ dependence of the order parameter $m_{q}^{*}$ at $\mu=0$ (Fig. 1) and $\mu=100,160,200,220 \mathrm{MeV}$ (Fig. 2). The numerical computation implemented for Eqs. (6) and (11) produces Figs. 1 and 2 with the main properties: Fig. 1 shows that at $\mu=0$ the order parameter decreases to zero as $T$ increases infinitely. Thus, the chiral symmetry gets restored and the behavior of the graph means that the transition is first order. Meanwhile, at non-vanishing $\mu$ Fig. 2 yields the expected scenario of chiral non-restoration. 


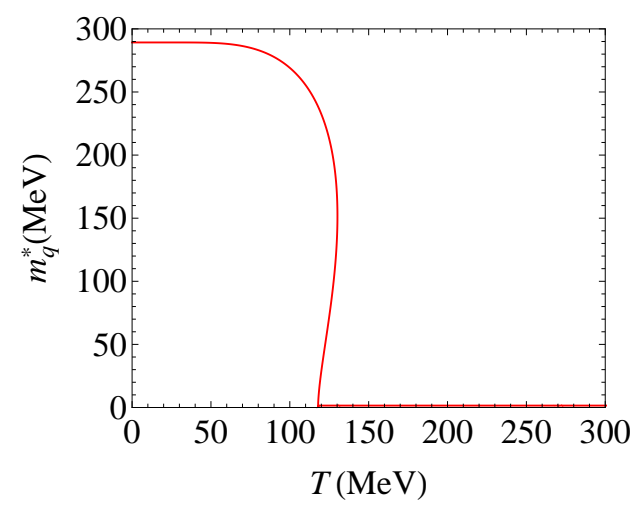

Fig. 1. The $T$ dependence of order parameter at $\mu=0$.

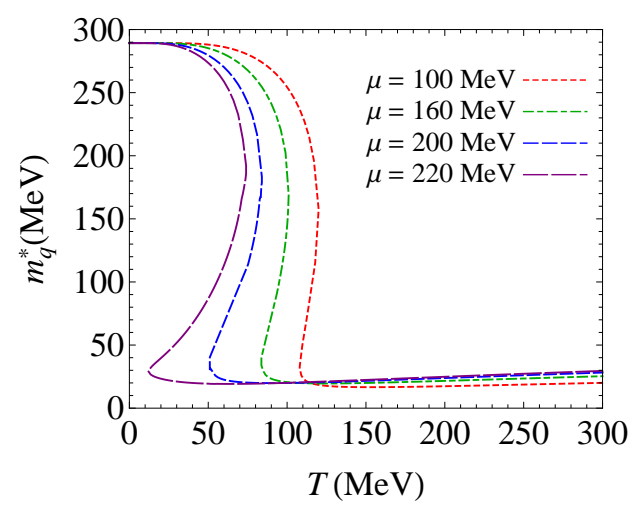

Fig. 2. The $T$ dependence of order parameter at $\mu=100 \mathrm{MeV}$ (dotted line), $160 \mathrm{MeV}$ (dashed-dotted line), $200 \mathrm{MeV}$ (dashed line) and $220 \mathrm{MeV}$ (long dashed line).

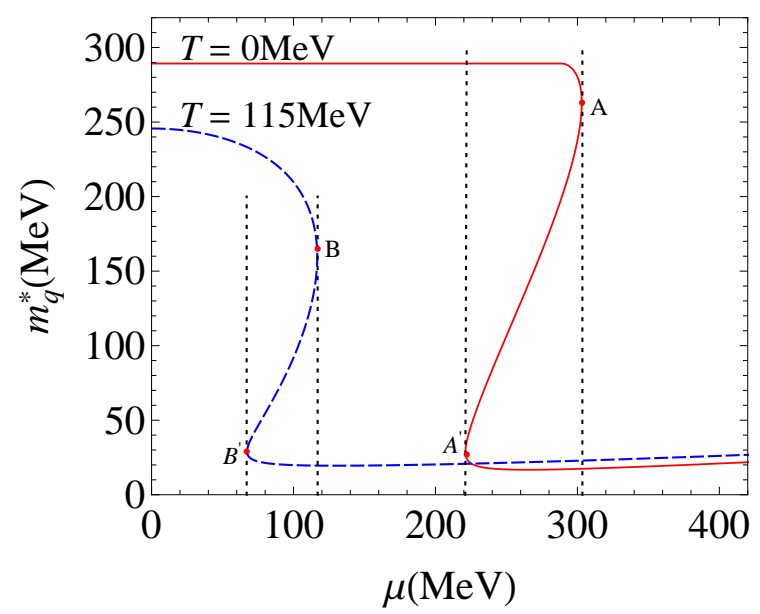

Fig. 3. The $\mu$ dependence of order parameter at $T=0$ (solid line), $115 \mathrm{MeV}$ (dashed line). The region of the first-order phase transition is restricted by two dotted lines. A, $\mathrm{A}^{\prime}$ and B , B' are isothermal spinodal points.

Next the $\mu$ dependence of order parameter $m_{q}^{*}$ at $T=0$ (solid line), $115 \mathrm{MeV}$ (dashed line) is plotted in Fig. 3. The behavior of $m_{q}^{*}$ typically describes the first - order phase transition in the region restricted by two dotted lines where it is a multi-valued function of $\mu$. The isothermal spinodal points which delimit the region of equilibrium first-order phase transition are denoted by A, A' and B, B'. It is easily seen that the chiral symmetry is not restored at high baryon density, too. As we will see later, the feature that $m_{q}^{*}$ is a multi-valued function of $\mu$ in a large range of $T$ gives rise to the specific behaviors of energy density and pressure as functions of $\mu$ in a large range of $T$. 


\section{LIFSHITZ PHASE TRANSITION}

It is commonly accepted that there exist so far two schemes of classifications relevant to physical systems:

a) The conventional classification by symmetry which reflects the phenomena of spontaneously broken symmetry when the energy is reduced.

b) Another scheme of classification deals with classifying vacuum states by momentumspace topology $[10,11]$. It reflects the opposite tendency, the symmetry gradually emerges at low energy. The universality classes of quantum vacuum states are determined by the momentum-space topology, which is primary while the vacuum symmetry is the emergent phenomenon in the low energy corner.

For all fermionic systems in three-dimensional (3D) space and invariant under translations of coordinates there are four basics universality classes of vacua provided by topology in momentum space $[10,11]$ :

- Vacua with fully-gapped fermionic energy excitations, such as semiconductors, superconductors and Dirac particles.

- Vacua with fermionic energy excitations characterized by Fermi points in 3D momentum space determined by $E(\vec{p})=0$.

- Vacua with fermionic energy excitations determined by Fermi surfaces in 3D momentum space, and

- Vacua with fermionic energy excitations characterized by lines in the 3D momentum space.

The phase transitions which follow from this classification scheme are quantum phase transition occurring at $T=0$. Among these QPT's the Lifshitz phase transition (LPT) is marked as a QPT from the fully-gapped state to the state with Fermi surface [12], which should be not confused with the Lifshitz points [13]. In recent years LPT becomes one of the hot topics in condensed matter physics [14-17]. The LPT in nuclear matter has been studied in Refs. $[18,19]$.

Now we present the LPT in the effective model (1) of QCD characterized by the chiral non-restoration at high temperature and/or baryon chemical potential. We first concern the case $T=0$, then extend to the finite temperature case.

In the vacuum state the quark energy excitation possesses the form (5),

$$
E_{ \pm}(\vec{p})=\sqrt{\vec{p}^{2}+m_{q}^{* 2}} \pm \mu^{*}
$$

Then the locus of Fermi points are determined by

$$
\begin{array}{ll}
E_{+}(\vec{p})=0 & \text { for } \mu^{*}>0 \\
E_{-}(\vec{p})=0 & \text { for } \mu^{*}<0
\end{array}
$$

Let us first consider the case $\mu^{*}>0$ where the locus of Fermi points in the $\left(p_{z}, \mu\right)$ plane is given by the equation

$$
p_{z}= \pm \sqrt{\mu^{* 2}-m_{q}^{* 2}}
$$




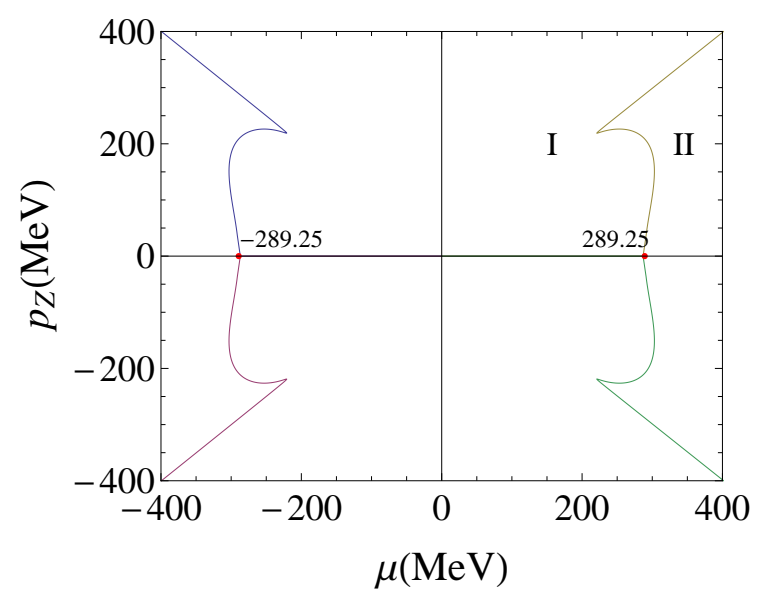

Fig. 4. The locus of Fermi points in the $\left(k_{z}, \mu\right)$ - plane in the case $\mu^{*}>0$.

In the Fig. 4 is shown this locus which is the boundary separating the domain I of fully-gapped vacua from the domain II of vacua with Fermi sphere, whose radius reads $p_{F}=\sqrt{\mu^{* 2}-m_{q}^{* 2}}$. The value $\mu_{0}=289.25 \mathrm{MeV}$, corresponding to $p_{F}=0$, is exactly the critical point of LPT because it separates the fully-gapped state, characterized by $\theta=\mu^{* 2}-m_{q}^{* 2}<0$, from the state with Fermi sphere, characterized by $\theta=\mu^{* 2}-m_{q}^{* 2}>0$, as $\mu$ changes from $\mu<289.25 \mathrm{MeV}$ to $\mu>289.25 \mathrm{MeV}$. LPT is actually controlled by the baryon chemical potential. For $\mu>289.25 \mathrm{MeV}$ there are two Fermi points in 3D momentum space which locate at the north and south poles, $N\left(0,0, p_{F}\right)$ and $S\left(0,0,-p_{F}\right)$, of a Fermi sphere. It is known that for a general system, relativistic or non-relativistic, the stability of the $j$-th Fermi point is guaranteed by the topological invariant, which is called the winding number $N_{j}$ defined as

$$
N_{j}=\frac{1}{24 \pi^{2}} \epsilon_{\mu \nu \rho \sigma} \operatorname{tr} \sum_{j} \oint d S^{\sigma} \frac{\partial}{\partial p_{\mu}} G^{-1} G \frac{\partial}{\partial p_{\nu}} G^{-1} G \frac{\partial}{\partial p_{\rho}} G .
$$

Here $G$ is the quark propagator, $\Sigma_{j}$ is the $2 \mathrm{D}$ surface around the $j$-th isolated Fermi point $E_{-}(\vec{p})=0, j=1,2$, and the trace is taken over all relevant indices. In our case

$$
G^{-1}=i \omega-H(\vec{p}), \quad H(\vec{p})=\vec{\alpha} \vec{p}+\mu^{*}-\beta m_{q}^{*}
$$

which, combining with (15) and (16), yields $N_{1}=+1, N_{2}=-1$. At $\mu=\mu_{0}$ these two points merge and form one topological trivial Fermi point $\vec{p}_{0}=0$ with winding number $N_{0}=N_{1}+N_{2}=0$. This intermediate state is marginal and cannot protect the vacuum against decay into two topologically stable vacua.

The stability of two Fermi points entails the stability of the Fermi sphere (FS) with poles $N$ and $S$. However, the Fermi sphere itself is also a topologically stable object protected by another winding number. Indeed, in the domain II means that

$$
E_{-} \approx \frac{\vec{p}^{2}-p_{F}^{2}}{2 \mu^{*}}=\frac{\vec{p}^{2}}{2 \mu^{*}}-\epsilon_{F}
$$




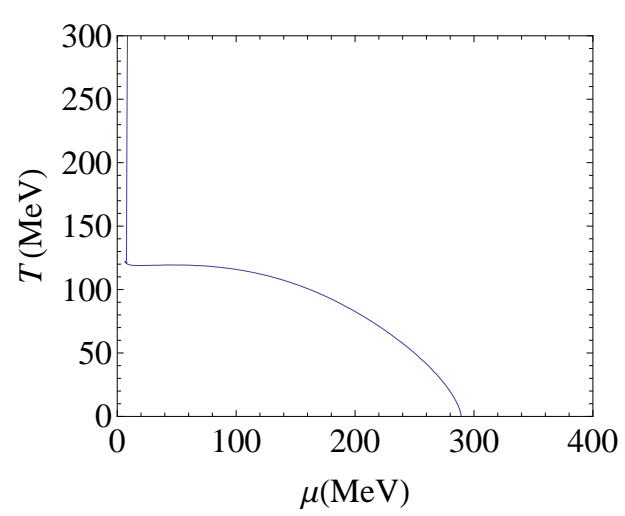

Fig. 5. Phase diagram of LPT.

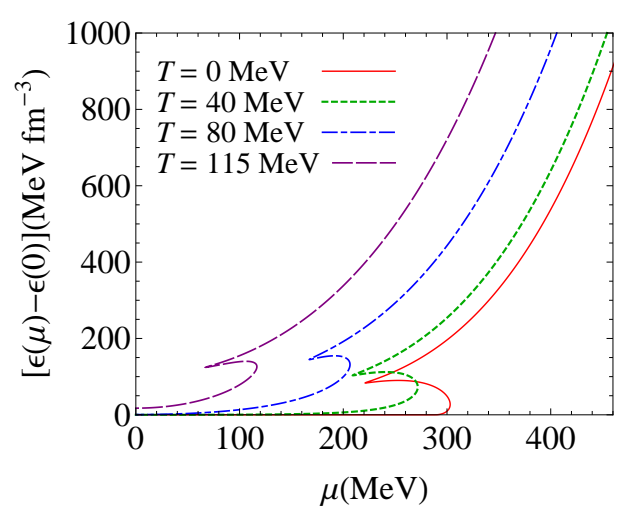

Fig. 6. The evolution of $\epsilon$ versus $\mu$ at $T=$ 0 (solid line), $40 \mathrm{MeV}$ (dotted line), $80 \mathrm{MeV}$ (dashed-dotted line) $115 \mathrm{MeV}$ (dashed line).

which leads to the inverse Green function (in imaginary frequency)

$$
G^{-1}=i \omega-\frac{\vec{p}^{2}}{2 \mu^{*}}+\varepsilon_{F}
$$

The stability of the Fermi sphere is then guaranteed by the non-vanishing winding number

$$
N_{F S}=\operatorname{tr} \sum_{\text {around FS }} \oint \frac{d p_{\mu}}{2 \pi i} G \frac{\partial}{\partial p_{\mu}} G^{-1}=1 .
$$

As for the energy excitation $E_{+}(\vec{p})$ the locus of Fermi points corresponds to $\mu<0$. As is shown in Fig. 4 the loci of Fermi points associating with $E_{+}(\vec{p})$ and $E_{-}(\vec{p})$ are symmetric through the $p_{z}$ axis. Hence, for the sake of simplicity we focus on the case $\mu>0$ only.

At finite temperature $m_{q}^{*}$ and $\mu^{*}$ turn out to be functions of both $T$ and $\mu$. As a matter of fact, due to (15) the states with Fermi sphere are determined by

$$
\theta(T, \mu)=\mu^{* 2}(T, \mu)-m_{q}^{* 2}(T, \mu)>0
$$

with two Fermi points

$$
p_{z}(T, \mu)= \pm \sqrt{\mu^{* 2}(T, \mu)-m_{q}^{* 2}(T, \mu)}
$$

The fully-gapped states are characterized by $\theta(T, \mu)<0$ and, consequently, the critical states are given by

$$
\theta(T, \mu)=\mu^{* 2}(T, \mu)-m_{q}^{* 2}(T, \mu)=0
$$

separating fully-gapped states from states with Fermi sphere. This means that $\theta$ plays the role of order parameter of LPT and Eq. (18) describes the phase diagram of LPT in the $(T, \mu)$-plane plotted in Fig. 5.

In order to determine the order of LPT let us examine the $\mu$ dependence of energy density, Eqs. (9) and (15), at several values of T. Its graphs depicted in Fig. 6 are typical for first-order phase transition: during phase transition the system emits an amount of 


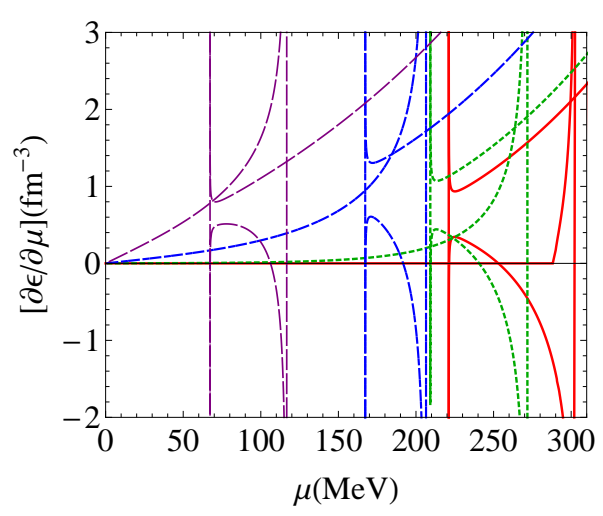

Fig. 7. The evolution of $\partial \epsilon / \partial \mu$ versus $\mu$ at $T=0$ (solid line), $40 \mathrm{MeV}$ (dotted line), $80 \mathrm{MeV}$ (dashed-dotted line) $115 \mathrm{MeV}$ (dashed line).

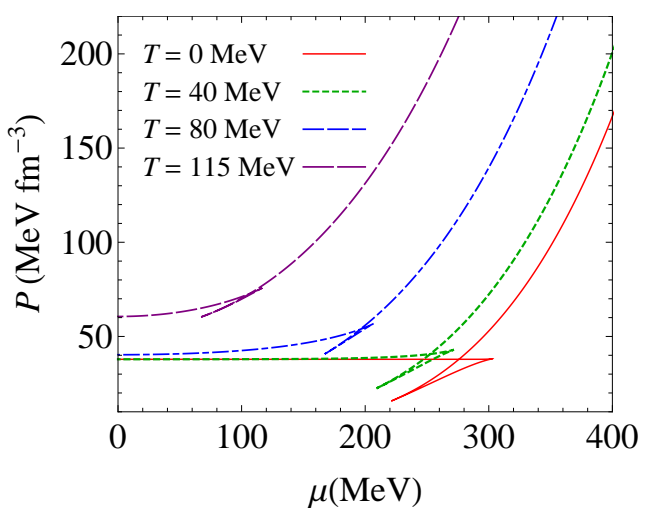

Fig. 8. The evolution of $P$ versus $\mu$ at $T=0$ (solid line), $40 \mathrm{MeV}$ (dotted line), $80 \mathrm{MeV}$ (dasheddotted line) $115 \mathrm{MeV}$ (dashed line).

latent heat. For example, at $T=0, \mu_{c}=289.25 \mathrm{MeV}$ the emitted amount of latent heat is equal to

$$
\Delta \epsilon=\epsilon(\mu=289.25 \mathrm{MeV}+0, T=0)-\epsilon(\mu=289.25 \mathrm{MeV}-0, T=0)=138.50 \mathrm{MeV}^{-3} \mathrm{fm} \text {. }
$$

Hence, we conclude that the LPT is first order.

In order to get a deeper insight into the issue let us observe simultaneously the evolutions of $\partial \epsilon / \partial \mu$ and the pressure $P$, Eqs. (8) and (13), versus $\mu$ at several temperature steps. The analytical expression of $\partial \epsilon / \partial \mu$ is given in Appendix. Their graphs are present in Fig. 7 and Fig. 8, from which it is easily recognized that these quantities have the same singular points in the $(T, \mu)$-plane.

Another feature of LPT is cleared up by investigating the evolution of quark density against $\mu$ at $T=0,40,80,115 \mathrm{MeV}$. Eqs. (7) and (12) together with the gap equation provide Fig. 9 which tells that at fixed $T$ as $\mu$ changes from $\theta(T, \mu)<0$ to $\theta(T, \mu)>0$ the system undergoes a transition from low density belonging to fully-gapped state from the low density fully-gapped state to high density state with Fermi surface. At $\theta(T, \mu)=0$ the system has a jump from low to high densities. If we identify these two states to the gas and liquid states then LPT describes a quantum liquid-gas phase transition of quark system.

To proceed to the stability of states in LPT let us introduce the density susceptibility defined as

$$
\chi=\frac{\partial \rho}{\partial \mu}=-\frac{\partial^{2} \Omega}{\partial \mu^{2}}
$$

whose analytical formula is presented in Appendix. It is commonly accepted that the density susceptibility is related to density fluctuation which allows us to observe signals of phase transition in experiments. We show in Fig. 10 the graphs describing the evolution of $\chi$ versus $\mu$ at $T=0,40,80,115 \mathrm{MeV}$. Let us analyze some characters exhibiting in this figure. For $T=0$, for example, we see that $\chi$ diverges at $\mu=302.4 \mathrm{MeV}$ and has three branches in the interval $250.10 \mathrm{MeV} \leq \mu \leq 302.40 \mathrm{MeV}$ : two positive branches 


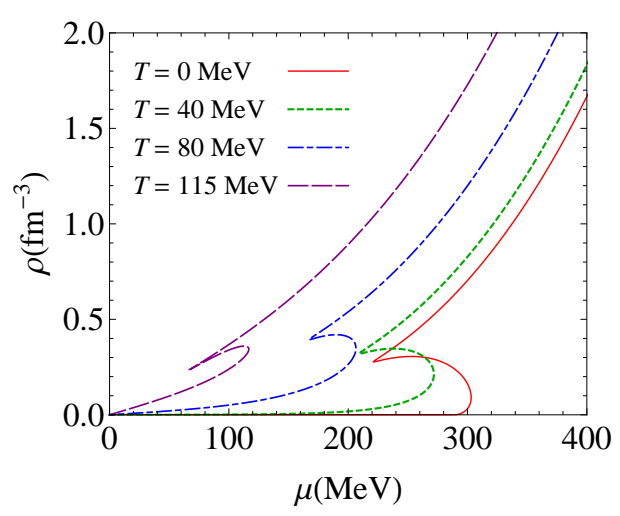

Fig. 9. The evolution of quark density versus $\mu$ at $T=0$ (solid line), $40 \mathrm{MeV}$ (dotted line), 80 $\mathrm{MeV}$ (dashed-dotted line) $115 \mathrm{MeV}$ (dashed line).

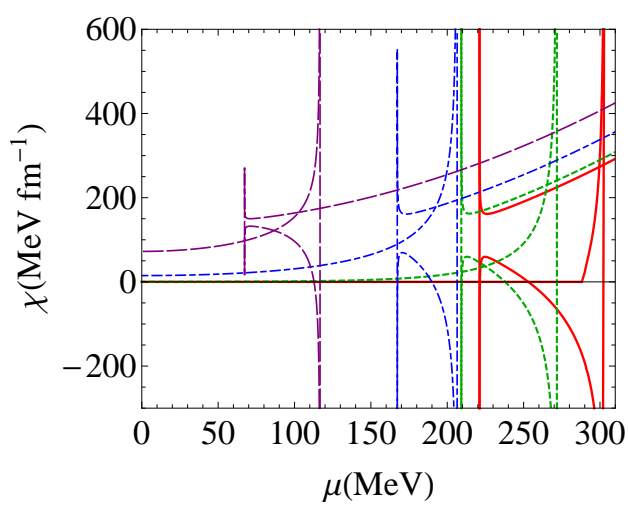

Fig. 10. The evolution of the density susceptibility versus $\mu$ at $T=0$ (solid line), $40 \mathrm{MeV}$ (dotted line), $80 \mathrm{MeV}$ (dashed-dotted line) 115 $\mathrm{MeV}$ (dashed line).

correspond to stable states which associate with minima of effective potential and the negative branch corresponds to mechanically unstable states where effective potential gets maximum, therefore, all points belonging to this branch describe the thermal spinodal line. The LPT occurring at $\mu_{0}=289.25 \mathrm{MeV}$ lies inside the region of mechanical unstability. At finite $T$ we have the same physical picture.

\section{CONCLUSION AND OUTLOOK}

In the preceding Section we presented the topological Lifshitz phase transition in an effective model of QCD where the chiral symmetry broken at $T=0$ never gets restored at high $T$ and/or $\mu$. The main results we found are in order

a) We indicated that under the LPT the quark system undergoes a transition from liquid to gas states which correspond respectively to fully-gapped state and state with Fermi sphere. In this connection, the gas state is stable under any small perturbation $[10,11]$ and the liquid state is protected by non-vanishing values of winding numbers.

b) We obtained the Lifshitz phase diagram in the $(T, \mu)$-plane.

c) We determined that the LPT takes place inside the region of mechanical unstability, within which the energy density and pressure manifest the singular behaviors.

It is easily recognized that what we mentioned above is valid for a large class of quark systems, whose quark energy excitation takes the form

$$
E=\sqrt{\vec{p}^{2}+M^{2}(T, \mu)}-F(T, \mu),
$$

$M$ and $F$ are functions of control parameter denoted by $\mu$ and other parameters involving temperature $T$. Then the order parameter of LPT is defined as

$$
\theta=F^{2}-M^{2} \text {. }
$$


Depending on $\mu$ the order parameter can get various values from $\theta>0$ to $\theta>0$. It results that corresponding to negative $\theta$ is the fully-gapped state and the positive $\theta$ corresponds to state with Fermi surface which is protected by momentum-space topology.

\section{ACKNOWLEDGMENTS}

T.H.P. is supported by the MOST through the Vietnam - France LIA collaboration. P.T.T.H and N.T.A. are supported by NAFOSTED under grant No. 103.01-211.05.

\section{REFERENCES}

[1] T. Hatsuda and K. Maeda, Quantum Phase Transition in Densed QCD, Chapter 25 of Developments in Quantum Phase Transitions, ed. by L. D. Carr (Taylor and Francis, 2010).

[2] S. Weinberg, Phys. Rev. D 9 (1974) 3357

[3] L. Dolan and R. Jackiw, Phys. Rev. D 9 (1974) 3357

[4] N. Schupper and N. M. Shnerb, Phys. Rev. E 72 (2005) 046107

[5] G. Bimonte and G. Lozano, Phys. Lett. B 366 (1996) 248.

[6] G. Dvali, A. Melfo and G. Senjanovic, Phys. Rev. Lett. 75 (1995) 4559. G. Dvali and G. Senjanovic, Phys. Rev. Lett. 74 (1995) 5178

[7] P. N. Meisinger and M. C. Ogilvie, Phys. Lett. B 379 (1996) 163

[8] S. Chandrasekharan and N. Christ, Nucl. Phys. B47, Proc. Suppl. (1996) 527

[9] J. B. Kogut and D. K. Sinclair, Phys. Rev. D 66 (2002) 034505

[10] G. E. Volovik, The Universe in a Helium Droplet, Clarendon, Oxford, 2003

[11] G. E. Volovik, Quantum Phase Transition from Topology in Momentum Space, Lecture Notes in Physics, Vol. 718 Springer, Berlin, 2007, p. 3

[12] I. M. Lifshitz, Sov. Phys. JETP 11 (1960) 1130

[13] E. M. Lifshitz, Zh. Eksp. Theor. Fiz. 11 (1941) 255

[14] N. Doiron-Leyrand et al., Nature (2007) 565

[15] D. Leboeuf et al., Nature 450, 533 (2007).

[16] Y. Yamaji, T. A. Misawa and M. Imada, Proc. Phys. Soc. Jpn. 75 (2005) 094719

[17] V. A. Khodel, J. W. Clark and M. V. Zverev, Phys. Rev. B 78 (2008) 075120

[18] Tran Huu Phat and Nguyen Van Thu, Phys. Rev. C 87 (2013) 024321

[19] Tran Huu Phat, Nguyen Tuan Anh and Phung Thi Thu Ha, Int. J. Mod. Phys. E 22 (2013) 1350077

\section{Appdendix. THE ANALYTICAL EXPRESSION FOR $\partial \epsilon / \partial \mu$ AND $\chi$}

$$
\frac{\partial \epsilon}{\partial \mu}=\frac{G B+H D}{B C+D E}, \quad \chi=\frac{A B+F D}{B C+D E}
$$


where

$$
\begin{aligned}
& A=\frac{2 m_{q}^{*}}{\pi^{2} T} I_{5}, \quad B=\frac{2 g_{v}^{2} m_{q}^{2}}{\pi^{4} T m_{\omega}^{2} m_{q}^{* 2}} I_{1} I_{3}-\frac{m_{q}^{* 2}}{2 \pi^{2} T} I_{5}, \quad C=\frac{4 g_{v}^{2} m_{q}^{2}}{\pi^{2} m_{\omega}^{2} m_{q}^{* 3}} I_{1}+\frac{2 g_{v}^{2} m_{q}^{2}}{\pi^{2} T m_{\omega}^{2} m_{q}^{*}} I_{5}, \\
& D=-\frac{m_{\sigma}^{2} m_{q}^{* 3}}{4 g_{s}^{2} m_{q}^{2}}+\frac{m_{\sigma}^{2} m_{q}^{*}\left(m_{q}^{2}-m_{q}^{* 2}\right)}{4 g_{s}^{2} m_{q}^{2}}+\frac{2 g_{v}^{2} m_{q}^{2}}{\pi^{4} m_{\omega}^{2} m_{q}^{* 3}} I_{1}-\frac{m_{q}^{*}}{2 \pi^{2}} I_{2}+\frac{m_{q}^{*}}{2 \pi^{2}} I_{9}-\frac{2 g_{v}^{2} m_{q}^{2}}{\pi^{4} T m_{\omega}^{2} m_{q}^{*}} I_{1} I_{5}, \\
& E=1+\frac{2 g_{v}^{2} m_{q}^{2}}{\pi^{2} T m_{\omega}^{2} m_{q}^{* 2}} I_{3}, \quad F=\frac{2}{\pi^{2} T} I_{3}, \\
& G=-\frac{m_{\sigma}^{2} m_{q}^{*}\left(m_{q}^{2}-m_{q}^{* 2}\right)}{2 g_{s}^{2} m_{q}^{2}}+\frac{4 g_{v}^{2} m_{q}^{2}}{\pi^{4} m_{\omega}^{2} m_{q}^{* 3}} I_{1}+\frac{2 m_{q}^{*}}{\pi^{2}} I_{2}-\frac{2 m_{q}^{*}}{\pi^{2} T} I_{3}-\frac{4 g_{v}^{2} m_{q}^{2}}{\pi^{4} T m_{\omega}^{2} m_{q}^{*}} I_{1} I_{5}, \\
& H=-\frac{4 g_{v}^{2} m_{q}^{2}}{\pi^{4} T m_{\omega}^{2} m_{q}^{* 2}} I_{1} I_{3}-\frac{2}{\pi^{2} T} I_{4}, \\
& P=-\frac{2 m_{q}^{*}}{\pi^{2} T} I_{8}, \quad Q=-\frac{2 g_{v}^{2} m_{q}^{2}}{\pi^{4} T m_{\omega}^{2} m_{q}^{* 2}} I_{1} I_{6}+\frac{m_{q}^{* 2}}{2 \pi^{2} T^{2}} I_{8}, \\
& K=\frac{2 g_{v}^{2} m_{q}^{2}}{\pi^{2} T^{2} m_{\omega}^{2} m_{q}^{* 2}} I_{6}, \quad N=-\frac{2}{\pi^{2} T} I_{6}, \quad M=\frac{2}{\pi^{2} T^{2}} I_{7},
\end{aligned}
$$

in which the integrals as follows,

$$
\begin{aligned}
I_{1} & =\int d k k^{2}\left(n_{+}-n_{-}\right), \\
I_{2} & =\int d k \frac{k^{2}}{E_{k}}\left(n_{+}+n_{-}\right), \\
I_{3} & =\int d k k^{2}\left(n_{+}+n_{-}-n_{+}^{2}-n_{-}^{2}\right), \\
I_{4} & =\int d k k^{2} E_{k}\left(n_{+}-n_{-}-n_{+}^{2}+n_{-}^{2}\right), \\
I_{5} & =\int d k \frac{k^{2}}{E_{k}}\left(n_{+}-n_{-}-n_{+}^{2}+n_{-}^{2}\right), \\
I_{6} & =\int d k k^{2}\left[E_{+}\left(n_{+}-n_{+}^{2}\right)-E_{-}\left(n_{-}-n_{-}^{2}\right)\right], \\
I_{7} & =\int d k k^{2}\left[E_{+}^{2}\left(n_{+}-n_{+}^{2}\right)+E_{-}^{2}\left(n_{-}-n_{-}^{2}\right)\right], \\
I_{8} & =\int d k \frac{k^{2}}{E_{k}}\left[E_{+}\left(n_{+}-n_{+}^{2}\right)+E_{-}\left(n_{-}-n_{-}^{2}\right)\right], \\
I_{9} & =\int d k \frac{k^{2}}{E_{k}^{2}}\left[\frac{m_{q}^{* 2}}{T}\left(n_{+}+n_{-}-n_{+}^{2}-n_{-}^{2}\right)-\frac{k^{2}}{E_{k}}\left(n_{+}+n_{-}\right)\right] .
\end{aligned}
$$

